\title{
B-physics from lattice QCD...with a twist
}

IFIC/12-80

FTUAM-12-113

IFT-UAM/CSIC-12-111

RM3-TH/12-20

LTH966

CERN-PH-TH/2012-328

N. Carrasco $(a)$, P. Dimopoulos ${ }^{(b, c)}$, R. Frezzotti ${ }^{(b, c)}$, V. Giménez $^{(a)}$, G. Herdoiza $^{(d)}$, V. Lubicz ${ }^{(e, f)}$, G. Martinelli ${ }^{(g, h)}$, C. Michael $^{(i)}$, D. Palao ${ }^{(c)}$, G.C. Rossi ${ }^{(b, c)}$, F. Sanfilippo ${ }^{(j)}$, A. Shindler ${ }^{* \dagger}(k)$, S. Simula ${ }^{(f)}$, C. Tarantino ${ }^{(e, f)}$

${ }^{(a)}$ Departament de Física Teòrica and IFIC, Univ. de València-CSIC

${ }^{(b)}$ Dipartimento di Fisica, Università di Roma "Tor Vergata"

(c) INFN, Sezione di "Tor Vergata" clo Dipartimento di Fisica, Universita ' di Roma "Tor Vergata"

${ }^{(d)}$ Departamento de Física Teòrica and Instituto de Física Teòrica UAM/CSIC,

${ }^{(e)}$ Dipartimento di Fisica, Università Roma Tre

${ }^{(f)}$ INFN, Sezione di Roma Tre clo Dipartimento di Fisica, Università Roma Tre

${ }^{(g)}$ SISSA

(h) INFN, Sezione di Roma,

${ }^{(i)}$ Theoretical Physics Division, Dept. of Mathematical Sciences, University of Liverpool

${ }^{(j)}$ Laboratoire de Physique Théorique (Bat. 210), Université Paris Sud,

${ }^{(k)}$ CERN, Physics Departement, Geneva

We present a precise lattice QCD determination of the $b$-quark mass, of the $B$ and $B_{s}$ decay constants and first results for the $B$-meson bag parameters. For our computation we employ the socalled ratio method and our results benefit from the use of improved interpolating operators for the $B$-mesons. QCD calculations are performed with $N_{f}=2$ dynamical light-quarks at four values of the lattice spacing and the results are extrapolated to the continuum limit. The preliminary results are $\bar{m}_{b}\left(\bar{m}_{b}\right)=4.35(12) \mathrm{GeV}$ for the $\overline{\mathrm{MS}} b$-quark mass, $f_{B_{s}}=234(6) \mathrm{MeV}$ and $f_{B}=197(10) \mathrm{MeV}$ for the $B$-meson decay constants, $B_{B_{s}}^{\bar{M} S}\left(\bar{m}_{b}\right)=0.90(5)$ and $B_{B}^{\bar{M} S}\left(\bar{m}_{b}\right)=0.87(5)$ for the $B$-meson bag parameters.

36th International Conference on High Energy Physics,

July 4-11, 2012

Melbourne, Australia

\footnotetext{
*Speaker.

${ }^{\dagger}$ Heisenberg Fellow
} 


\section{Introduction}

The search for New Physics (NP) together with stringent tests of the Standard Model (SM) pass through a detailed study of physical processes involving the $b$-quark. Two particularly important cases for the detection of potentially large NP contributions are the purely leptonic decays $B \rightarrow \tau v_{\tau}$ and $B_{s} \rightarrow \mu^{-} \mu^{+}{ }^{1}$ The relevant entries in the SM prediction for these decay rates are the CKM matrix element $V_{u b}$, and the pseudoscalar decay constants $f_{B}$ and $f_{B_{s}}$. Additionally the B-Physics parameters, $\Delta m_{d} / \Delta m_{s}, \Delta m_{d}$, play a crucial role in the Unitarity Triangle Analysis, and their determination rely on lattice QCD computations of the bag-parameters $B_{B_{s}}$ and $B_{B_{s}} / B_{B_{d}}$.

Despite the present (B-factories, LHCb) and future (SuperB factories) experimental programs, lattice QCD results of hadronic parameters need to have reduced uncertainties at the level of $\sim 1 \%$ (see refs [2, 3] for recent reviews).

In this proceeding we report on the ongoing project, within the ETMC collaboration, to compute B-physics hadronic parameters with Wilson twisted mass (Wtm) lattice fermions. We extend and improve our previous analysis $[4,5]$ in two ways:

- we optimize the interpolating operators for heavy-light systems to better project onto the fundamental state

- we extend the range of heavy masses, $\mu_{h}$, considered reaching values of approximately $\mu_{h} \sim$ $2.5 m_{c}$, where $m_{c}$ is the charm quark mass.

\section{Computational details}

We use, for this analysis, the $N_{f}=2$ dynamical gauge configurations with up and down mass degenerate quarks, $m_{u / d}=\mu_{l}$, generated by the European Twisted Mass Collaboration (ETMC) [6]. The lattice action is the tree-level improved Symanzik gauge action [7] and the twisted mass quark action [8] at maximal twist [9]. The strange and the charm quarks are quenched in this work.

We have used four lattice spacings [10] and we take the values of the light and strange renormalized quark masses together with the pseudoscalar density renormalization constants $Z_{P}^{\overline{\mathrm{MS}}}(2 \mathrm{GeV})$ respectively from [10] and [11]. All the lattice details of our computation can be found in our recent lattice 2012 contribution [12]. As usual heavy-light meson masses and decay constants are extracted studying the Euclidean time dependence of the 2-point functions with heavy-light interpolating operators. To improve the projection onto the fundamental state and keep the noise-to-signal ratio under control we extract meson masses at relatively small temporal separations. Thus the lowest lying energy eigenstate must have a large overlap with our interpolating fields. We have constructed these fields using the so-called Gaussian smearing [13] (see [12] for details). The improvement with respect with the standard local interpolating operators can be seen in the left plot of fig. 1 where we show the Euclidean time dependence of the effective masses for a particular simulation point obtained from local-local (LL), and two improved interpolating operators. The plot describes well the general properties for heavy-light effective masses: the improved correlators allow a safe extraction of the ground state mass at shorter Euclidean time separations compared to the LL correlators.

\footnotetext{
${ }^{1}$ Very recently the first measurement of this decay rate [1] shows a good agreement with the SM prediction.
} 

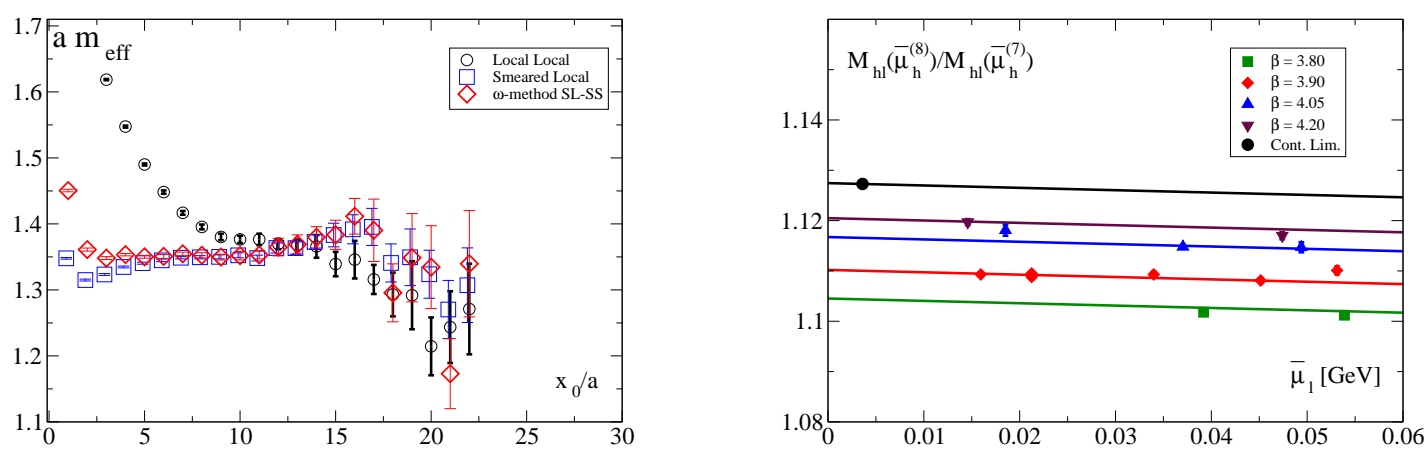

Figure 1: Left plot: Euclidean time dependence of the effective masses at a lattice spacing of $a=$ 0.098(3) fm, of a heavy-light correlation function obtained from LL and improved correlation functions. The heavy-quark mass corresponds to $\sim 2.3 m_{c}$. Right plot: chiral-continuum extrapolation of the ratio of heavy-light meson masses at the heaviest quark masses, $\bar{\mu}_{h}^{(7,8)}$.

\section{The $b$-quark mass and decay constants $f_{B}$ and $f_{B_{s}}$}

To determine the B-physics quantities we implement the ratio-method (see ref. [5] for the details of the method). We shortly recall here the basic idea for the computation of the $b$-quark mass. The same strategy applies for the other quantities computed in these proceedings. The static limit of the heavy-light meson mass $M_{h l}$ in the pole heavy-quark mass $\mu_{h}^{\text {pole }}$ is given by

$$
\lim _{\mu_{h}^{\text {pole }} \rightarrow \infty}\left(M_{h l} / \mu_{h}^{\text {pole }}\right)=1 .
$$

We take now a set of heavy-quark masses $\bar{\mu}_{h}^{(1)}<\bar{\mu}_{h}^{(2)}<\cdots<\bar{\mu}_{h}^{(N)}$ with ratio $\lambda$, i.e. $\bar{\mu}_{h}^{(n)}=\lambda \bar{\mu}_{h}^{(n-1)}$, fixed. We denote the masses renormalized in the $\overline{\mathrm{MS}}$ at a $2 \mathrm{GeV}$ scale with a "bar". The ratios of heavy-light meson masses at subsequent values of the heavy-quark mass, $y\left(\bar{\mu}_{h}^{(n)}, \lambda ; \bar{\mu}_{l}, a\right)$, properly normalized, are the key quantities of the ratio-method [5]. From QCD asymptotic freedom and eq. (3.1) it follows that the ratio $y$ in the continuum limit

$$
\lim _{\bar{\mu}_{h} \rightarrow \infty} \lim _{a \rightarrow 0} y\left(\bar{\mu}_{h}^{(n)}, \lambda ; \bar{\mu}_{l}, a\right)=1,
$$

has an exact static limit. The chiral and continuum extrapolation of the ratio of the heavy-light meson masses evaluated at the heaviest quark mass is shown in the right plot of fig. 1. The lattice results are well described by a fit linear in $a^{2}$ and in the light quark mass. We also note that discretization errors are sufficiently well under control. As it can be seen from the left plot in fig. 2, the ratio $y\left(\bar{\mu}_{h}, \lambda\right)$, extrapolated to the chiral and continuum limit, has a non-perturbative heavy-quark mass dependence that is well described by the HQET-inspired function

$$
y\left(\bar{\mu}_{h}, \lambda\right)=1+\frac{\eta_{1}(\lambda)}{\bar{\mu}_{h}}+\frac{\eta_{2}(\lambda)}{\bar{\mu}_{h}^{2}} .
$$

This plot clearly shows that the accurate description of the non-perturbative dependence of the ratio $y$ on $\bar{\mu}_{h}$ is possible only because of the improvements that we have implemented in this analysis. In particular the results are well described by the fit ansatz in eq. (3.3), that provides, with the fit parameters $\eta_{1}$ and $\eta_{2}$, the non-perturbative description of the heavy-quark mass dependence of the 

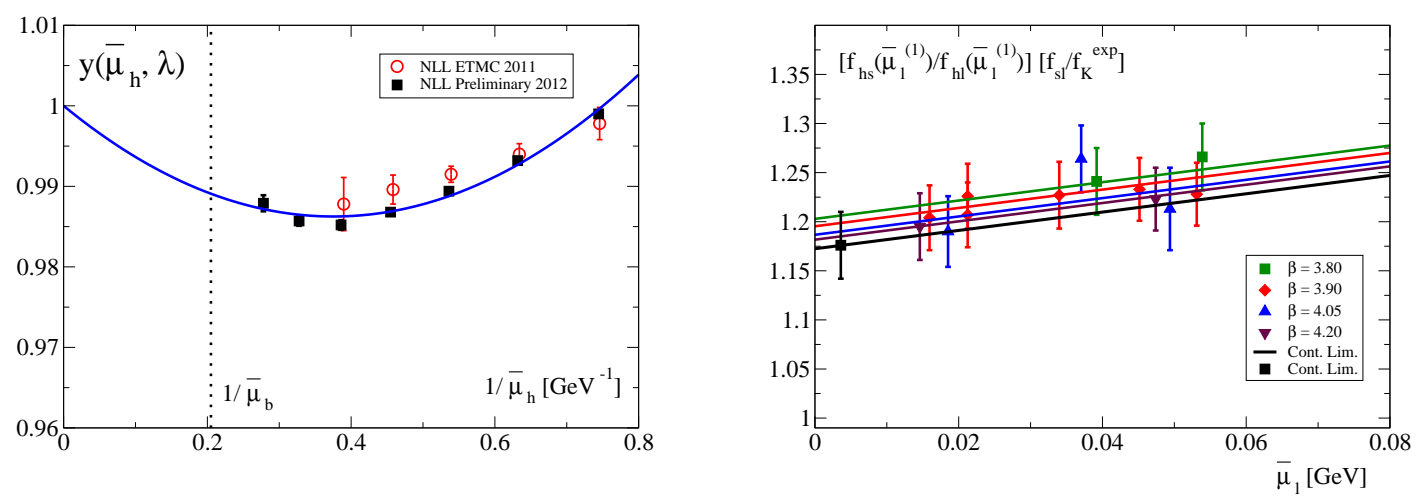

Figure 2: Left plot: the continuum ratio-function $y\left(\bar{\mu}_{h}, \lambda\right)$, for $\lambda=1.1784$ as a function of the heavy-quark mass. For comparison we show our previous results [4] without the interpolating operators improvement. Right plot: chiral extrapolation for the double-ratio $\left[f_{h s} / f_{h l}\right] \cdot\left[f_{s l} / f_{K}^{\exp }\right]$ at the triggering point, $\bar{\mu}_{h}^{(1)}$.

ratio $y\left(\bar{\mu}_{h}, \lambda\right)$ over the whole range of heavy-masses. The $b$-quark mass can be computed simply taking the triggering heavy-light meson mass $M_{h l}\left(\bar{\mu}_{h}^{(1)}\right)$ around the charm region and through a repeated application of the ratio $y\left(\bar{\mu}_{h}, \lambda\right)$ reaching the experimental value of the $B$ meson mass $M_{B}$. This is always possible with a slight tuning of $\lambda$ and $\bar{\mu}_{h}^{(1)}$ that leads after a 4-loop evolution to ${ }^{2}$

$$
\left.m_{b}^{\overline{M S}}\left(m_{b}\right)\right|_{N_{f}=2}=4.35(12) \mathrm{GeV} .
$$

We obtain a perfectly consistent result if we use as input the heavy-strange meson mass $M_{h s}$. The result we present here (3.4) is in agreement with our previous determination [4] with slightly reduced error. The $2.7 \%$ relative error is dominated by the ones associated to the lattice spacing and $Z_{P}$ determinations. The statistical and systematic uncertainties coming from the application of the ratio method turn out to be negligible, after the improvements just discussed.

A completely analogous strategy can be adopted to compute heavy-light and heavy-strange decay constants. We find advantageous to define ratios with exactly known static limit for the heavystrange decay constant, $z_{s}\left(\bar{\mu}_{h}^{(n)}, \lambda ; \bar{\mu}_{l}, \bar{\mu}_{s}, a\right)$, and for the ratio $f_{B_{s}} / f_{B}, \zeta\left(\bar{\mu}_{h}^{(n)}, \lambda ; \bar{\mu}_{l}, \bar{\mu}_{s}, a\right)$ [4]. Both $f_{h s}\left(\bar{\mu}_{h}^{(1)}\right)$ at the triggering point and the ratio $z_{s}$ have a smooth chiral-continuum extrapolation with cutoff effects always well under control. The heavy-quark mass dependence in the continuum of $z_{s}$ can again be described by a formula as the one in eq. (3.3). We use the $b$-quark mass, $\bar{m}_{b}$, we have determined as input leading us, using the ratio-method, to

$$
f_{B_{s}}=234(6) \mathrm{MeV} \text {. }
$$

The double-ratio $\zeta\left(\bar{\mu}_{h}^{(n)}, \lambda ; \bar{\mu}_{l}, \bar{\mu}_{s}, a\right)$ has a very smooth and weak dependence on the lightquark mass, the lattice spacing and the heavy-quark mass. The chiral behaviour of $f_{h s} / f_{h l}$ at the triggering mass, $\bar{\mu}_{h}^{(1)}$, suggests that the heavy-quark still behaves as a relativistic one. Thus for the chiral-continuum extrapolation of $f_{h s} / f_{h l}\left(\bar{\mu}_{h}^{(1)}\right)$ we find convenient to study the double-ratio $\left[f_{h s} / f_{h l}\right] \cdot\left[f_{s l} / f_{K}^{\mathrm{exp}}\right]$, for which a smooth dependence on the light quark mass is expected $[14,15]$. The results are well described by a linear fit in the light-quark mass as it can be seen from the right

\footnotetext{
${ }^{2}$ Note that a $b$-quark mass value smaller by $\sim 120 \mathrm{MeV}$ would be found by setting $N_{f}=4$ (rather than 2 ) in the evolution from $2 \mathrm{GeV}$ to the $b$-scale.
} 
plot in fig. 2. This analysis of $\zeta\left(\bar{\mu}_{h}^{(n)}, \lambda ; \bar{\mu}_{l}, \bar{\mu}_{s}\right)$ and $\left[f_{h s} / f_{h l}\right] \cdot\left[f_{s l} / f_{K}^{\text {exp }}\right]$ leads to the values

$$
\frac{f_{B_{s}}}{f_{B}}=1.19(5), \quad f_{B}=197(10) \mathrm{MeV}
$$

where we add in quadrature the statistical and systematic uncertainties.

\section{Bag parameters}

The renormalized operator $O_{q}^{\Delta B=2} \equiv\left[\bar{b} \gamma_{\mu}\left(1-\gamma_{5}\right) q\right]\left[\bar{b} \gamma_{\mu}\left(1-\gamma_{5}\right) q\right]$ in QCD is parametrized in terms of the bag parameter $B_{B_{q}}(\mu)$ as follows

$$
\left\langle\bar{B}_{q}\left|O_{q}^{\Delta B=2}\right| B_{q}\right\rangle^{\overline{M S}} \equiv \frac{8}{3} f_{B_{q}}^{2} B_{B_{q}}(\mu) M_{B_{q}}^{2}
$$

where $\mu$ is the renormalization scale. With Wtm fermions one can define an operator with the correct continuum limit whose renormalization is multiplicative [16]. Using heavy-quark symmetry one can show that the ratio of renormalized $B$-parameters evaluated in QCD is expected to approach unity as $1 / \bar{\mu}_{h} \rightarrow 0$, with corrections of order $1 / \log \left(\bar{\mu}_{h} / \Lambda_{Q C D}\right)$ for small values of $1 / \bar{\mu}_{h}$. To analyze the impact of such corrections we consider the ratio

$$
\omega_{q}\left(\bar{\mu}_{h}, \lambda ; \bar{\mu}_{l}, a\right)=\frac{B_{B_{q}}\left(\bar{\mu}_{h}, \bar{\mu}_{l}, a ; \mu\right)}{B_{B_{q}}\left(\bar{\mu}_{h} / \lambda, \bar{\mu}_{l}, a ; \mu\right)} \cdot \frac{C\left(\bar{\mu}_{h} ; \mu^{*}, \mu\right)}{C\left(\bar{\mu}_{h} / \lambda ; \mu^{*}, \mu\right)},
$$

where the $C$-factors ratio contains the info on the $1 / \log \left(\bar{\mu}_{h}\right)$-corrections at a fixed order in renormalization group (RG) improved perturbation theory (PT). Matching HQET to QCD in PT one can evaluate the function $C$. We consider here HQET-to-QCD matching only at tree level and LL order in PT (thereby avoiding the complications of operator mixing in HQET [17]), and confirm the impact of $1 / \log \left(\bar{\mu}_{h}\right)$-corrections on the final results to be at the level of one standard deviation [18]. The chiral and continuum limit of the ratio $\omega_{s}$ at the heaviest mass is well under control, see left plot of fig. 3. We refer to our contribution [18] to the lattice 2012 conference for additional details. The heavy-quark mass dependence of $\omega_{s}$ is well described by the formula $\omega_{s}\left(\bar{\mu}_{h}\right)=1+c_{1}(\lambda) / \bar{\mu}_{h}+c_{2}(\lambda) / \bar{\mu}_{h}^{2}$, see right plot of fig. 3. Applying the ratio-method one finally gets

$$
B_{B_{s}}^{\bar{M} S}\left(\bar{m}_{b}\right)=0.90(5)
$$

where the error is the sum in quadrature of statistical and systematic uncertainties. If we apply the ratio-method to $B_{B_{d}}$ and $B_{B_{s}} / B_{B_{d}}$ we obtain the preliminary estimate of

$$
\frac{B_{B_{s}}}{B_{B_{d}}}=1.03(2), \quad B_{B_{d}}^{\overline{M S}}\left(\bar{m}_{b}\right)=0.87(5) .
$$

Given this result we can also give a preliminary estimate for the parameters

$$
f_{B_{s}} \sqrt{B_{B_{s}}^{\mathrm{RGI}}}=274(10) \mathrm{MeV}, \quad f_{B} \sqrt{B_{B}^{\mathrm{RGI}}}=226(14) \mathrm{MeV}, \quad \xi=\frac{f_{B_{s}} \sqrt{B_{B_{s}}}}{f_{B} \sqrt{B_{B}}}=1.21(6),
$$

where the errors are the sum in quadrature of all uncertainties. 

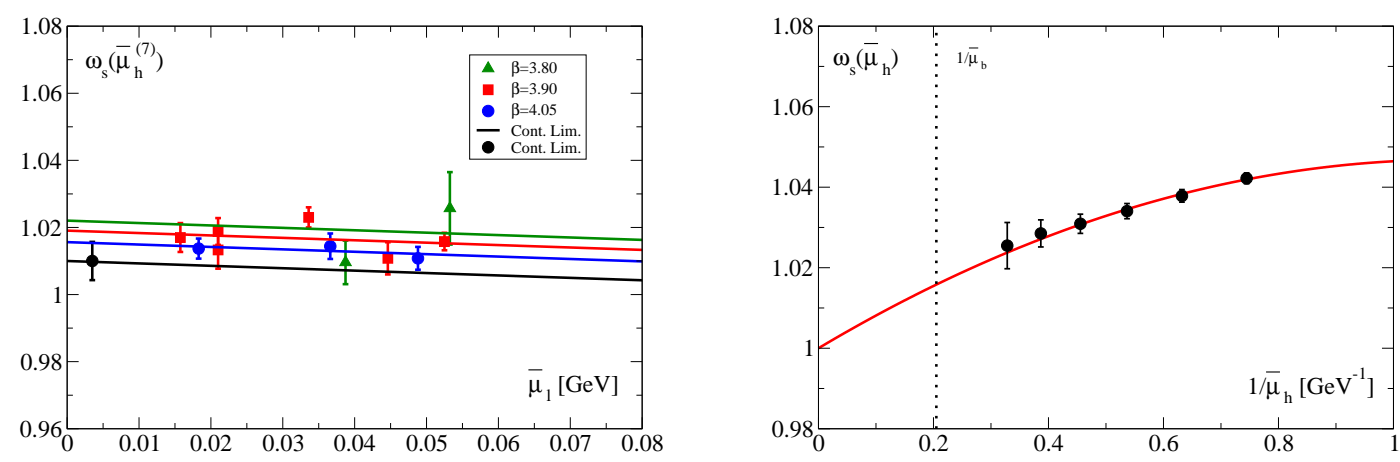

Figure 3: Left plot: chiral-continuum extrapolation of the ratio of the bag parameters, $\omega_{s}$, for the heaviest quark mass analyzed, $\bar{\mu}_{h}^{(7)}$. Right plot: heavy-quark mass dependence of the ratio function $\omega_{s}\left(\bar{\mu}_{h}, \lambda\right)$, for $\lambda=1.1784$ in the continuum and its non-perturbative description.

\section{Acknowledgements}

The computer time was made available to us by the Italian SuperComputing Resource Allocation (ISCRA) under the class A project HP10A7IBG7 "A New Approach to B-Physics on Current Lattices" and the class C project HP10CJTSNF "Lattice QCD Study of B-Physics" at the CINECA supercomputing service. We also acknowledge computer time made available to us by HLRN in Berlin.

\section{References}

[1] LHCb Collaboration, R. Aaij et al., (2012), 1211.2674.

[2] C. Tarantino, (2012), 1210.0474.

[3] J. Zanotti, PoS ICHEP2012 (2012) 036.

[4] ETM Collaboration, P. Dimopoulos et al., JHEP 1201 (2012) 046, 1107.1441.

[5] ETM Collaboration, B. Blossier et al., JHEP 1004 (2010) 049, 0909.3187.

[6] ETM Collaboration, R. Baron et al., JHEP 1008 (2010) 097, 0911.5061.

[7] P. Weisz, Nucl.Phys. B212 (1983) 1.

[8] Alpha collaboration, R. Frezzotti et al., JHEP 0108 (2001) 058, hep-lat/0101001.

[9] R. Frezzotti and G.C. Rossi, JHEP 0408 (2004) 007, hep-lat/0306014.

[10] ETM Collaboration, B. Blossier et al., Phys.Rev. D82 (2010) 114513, 1010.3659.

[11] ETM Collaboration, M. Constantinou et al., JHEP 1008 (2010) 068, 1004.1115.

[12] N. Carrasco et al., PoS LATTICE2012 (2012) 104, 1211.0568.

[13] S. Gusken, Nucl.Phys.Proc.Suppl. 17 (1990) 361.

[14] A. Roessl, Nucl.Phys. B555 (1999) 507, hep-ph/9904230.

[15] RBC-UKQCD Collaboration, C. Allton et al., Phys.Rev. D78 (2008) 114509, 0804.0473.

[16] R. Frezzotti and G.C. Rossi, JHEP 0410 (2004) 070, hep-lat/0407002.

[17] D. Becirevic et al., JHEP 0408 (2004) 022, hep-lat/0401033.

[18] ETM collaboration, N. Carrasco et al., (2012), 1211.0565. 